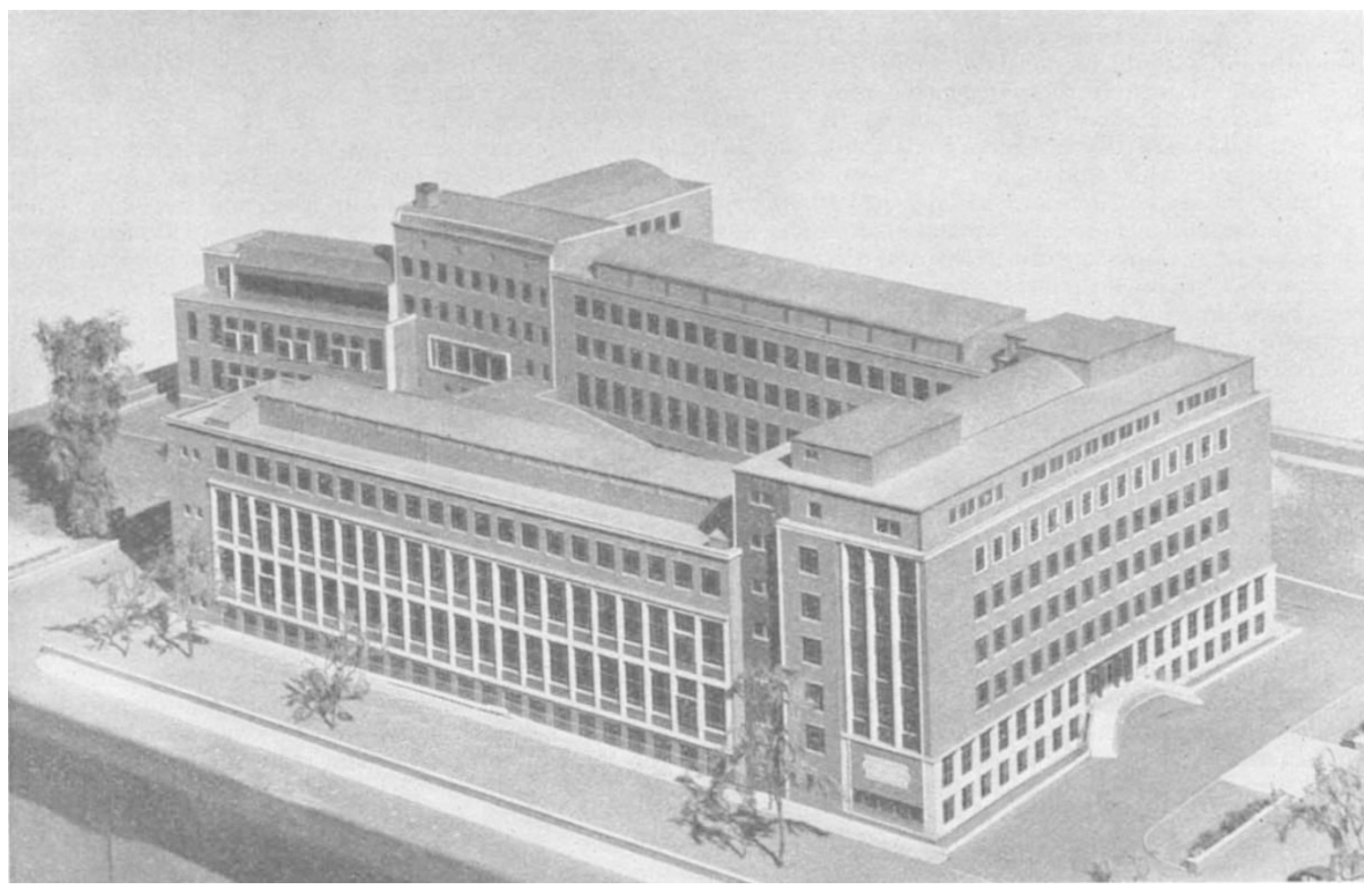

Fig. 1. University Chemical laboratory, Cambridge. Photograph of the complete model of the Laboratory, viewed from the northFig. 1. University Chemical Laboratory, Cambridge. Photograph of the complete model of the the Department of Physical chemistry,
west. The main entrance is shown on the right. The long bloek in the foreground is occupied by the
and that in the background by the Department of Organic and Inorganic Chemistry: the lecture rooms lie between these blocks. The two smaller blocks at the far left-hand corner are still under construction

lattice girders, and which are coated internally with acid-resisting plastic paint. Hot effluent from steam outlets is, however, carried through intermediate lead or stoneware piping.

To allow the desired flexibility in the size of the research laboratories, the teak bench tops are supported on cantilever brackets which fit into service racks designed to carry the tops at sitting or standing height, or to enable them to be completely removed. Independent cupboards of standard size but of various internal designs fit snugly beneath the bench tops : a research worker can thus choose a selection of cupboards suitable for his own work.
The design and construction of this Laboratory have presented many difficult and often novel problems. The University is particularly indebted to Mr. J. Murray Easton and Mr. S. E. T. Cusdin, of Messrs. Easton and Robertson, the architects, and to Mr. A. R. Gilson, the former superintendent of the Laboratory, who has closely and very effectively co-operated with them for the success of this project, which, in the hands of the contractors, Messrs. Kerridge and Co. (Cambridge), Ltd., and Messrs. Rattee and Kett, Ltd., has provided a chemical laboratory in which the University may well take great pride.

\title{
COSMIC RAYS
}

\section{CONFERENCE AT DURHAM}

\begin{abstract}
A PHYSICAL Society Conference on "Cosmic A Rays" was held in the Durham Colleges in the University of Durham on September 24 and 25. About one hundred delegates from most of the European centres of cosmic ray rosearch gathered to discuss topics ranging from the cosmological significance of large extensive air showers to recent measurements of the cosmic ray intensity in deep Canadian mines.

The theme of the Conference was two-fold: the cosmic and geophysical significance of cosmic ray measurements, and the nuclear physics of singleparticle interactions. The Conference was divided into short sessions in which an invited review paper
\end{abstract}

of $45 \mathrm{~min}$. was followed by a number of $10-\mathrm{min}$. papers.

The first session was devoted to a discussion of interactions in the energy-range $1-100 \mathrm{GeV}$. Recent results with machines were considered at the lower end of the energy region and the results of cosmic ray proton experiments for the remainder. Experimentally, several features appear to be clear: the inelasticity of proton interactions with light elements does not vary greatly with proton energy; the value is about 60 per cent, and the rate of production of heavy mesons increases slowly with increasing energy. Also clear is the inadequacy of the various theories of meson production-Fermi, Landau, etc.- to account 
for the observed angular distribution of meson production in the centre of mass system. The experimental results would be consistent with the isotropic emission of mesons from two centres moving away from the collision more slowly than do the original nucleons, but there does not seem to be any theoretical justification for this model. Since it is unlikely that machines capable of accelerating protons to energies much above $20 \mathrm{GeV}$. will be constructed in the next few years, attention is naturally focused on the cosmic ray experiments in this energy region. At present the interpretation of the experiments is difficult: the momenta of the primary protons are not measured, in fact, often the directions are not determined, and the secondary momenta are too high for accurate measurements. This point was well brought out in the first contribution, which described the results of a design study for an experiment in which accurate measurements of the parameters of the interaction would be possible. Such an experiment would be expensive by cosmic ray standards but not by machine standards ; it is perhaps within the scope of a collaborate university venture.

In the second session the energy-range was extended to $100,000 \mathrm{GeV}$. Most of the information in this region has come from studies of the so-called 'jets' observed in nuclear emulsions exposed in high-flying aircraft and balloons. The number of particles produced in the cascade resulting from some of the very energetic nuclear encounters (above a few thousand $\mathrm{GeV}$.) is so great that the events can be seen in the processed emulsion with the naked eye. At these high energies there is quite copious production of particles heavier than the pion, for example, the ratio of the charged heavy particles to all charged particles is $18+5$ per cent. Another important result is that the interaction length of the secondaries of mean energy around $200 \mathrm{GeV}$. is very close to that found for pions and protons in the region of a few $\mathrm{GeV}$. This result has important implications in the study of the development of the various components of the cosmic radiation through the atmosphere. Thus, for example, the pion flux is subject to the competing mechanisms of decay and interaction and is consequently sensitive to the value of the interaction length.

The energy-range under review was extended still further in the next session when various aspects of extensive air showers were discussed. The cosmological aspect is of supreme interest, bearing as it does on the origin of the cosmic radiation. The situation here has not changed over the past few years: the search for anisotropies in the primary cosmic radiation at energies up to $10^{18} \mathrm{eV}$. or so has still failed to produce statistically significant results. The need at present is for an order of magnitude increase in the size of the detecting arrays for extensive shower studies, and at least two such projects are afoot at the present time. Even with improved experimentation, however, the interpretation of the sea-level shower data is not straightforward. The electron component, which is traditionally measured, is subject to considerable fluctuations, arising in the main from the fluctuation in the height of the first primary proton interaction. For this reason attention is now being turned to the muon component for which the fluctuations are smaller, although difficulty is encountered due to the particle densities being considerably lower, at least for distances less than a fow hundred metres from the shower core. The session finished with the description of a number of new techniques by which detectors of the necessary large area could be constructed.

The current interest in the International Geophysical Year was reflected by a session on cosmic rays and solar-terrestrial relations. A considerable mass of data is accumulating on this subject from measurements or the variation with time of the various components, particularly the neutron component. The spectacular increases in intensity during the big solar flares has emphasized the part played by the Sun in producing low-energy cosmic rays. There is as yet no completely satisfactory explanation of the way in which these particles are accelerated or of the modulation of the primary cosmic rays by the Sun's magnetic field, but the result of the analysis of the very detailed International Geophysical Year results may lead to a considerable advance.

The interaction of the primary cosmic rays with the Earth's magnetic field was discussed by speakers from the Imperial College of Science and Technology. Experiments over the past few years have shown that the position of the cosmic ray equator, that is, the locus of the minimum of the cosmic ray intensity, is not consistent with the magnetic equator based on an eccentric dipole field. The disagreement amounts to a westward displacement in longitude of about $45^{\circ}$, and an attractive explanation was the bending of the lines of force from the rotating Earth by a surrounding region of highly ionized interplanetary matter. The Imperial College group has shown, however, that the dipole approximation is not a good one even at the distance from the Earth where most of the magnetic deflexion of the primary cosmic ray takes place. A more accurate treatment of the effect of the Earth's field taking into account the various magnetic anomalies removes the discrepancy between the respective equators.

The development of the muon component through the atmosphere and below ground occupied the next session. It was demonstrated that measurements on the sea-level muon flux give information on the multiplicity and mass spectrum of the products of primary interactions. Comparison with the underground depth-intensity relation gives information on the energy losses of muons of energy up to 1,000 $\mathrm{GeV}$.--information that is not available from other measurements.

With the introduction of new techniques of measurement, in particular the neon flash-tube of Conversi, and the consequent ease of measurement of high particle momenta, there has been something of a revival of muon spectrum measurements. Large magnetic spectrographs have been completed recently at sea-level at Cornell and Durham, and a number of smaller instruments are under construction for measurements of muon spectra in inclined directions and at various depths underground. The next few years should see significant advances in this subject.

The interactions of individual fast muons with nuclei were also discussed in this session. This interaction is via the flux of virtual photons accom panying the muon, and recent advances have been made both in the theoretical study and the experimental determination of cross-sections and other parameters of the interactions. Although there is still some slight doubt as to the way in which the original Williams-Weizsäcker treatment of the virtual photon flux is to be modified for ultra high-energy muons, the main features, such as the angular deflexions of the interacting muons and the multiplicity of the pion secondaries, are understood. The impor- 
tance of these measurements lies in the fact that what is being studied is effectively the interaction of photons of energies much above those available from artificial sources. The most that can be said about the variation of photo-nuclear cross-section with energy at the moment is that there is no strong evidence to suggest a cross-section varying very much from $10^{-28} \mathrm{~cm} \cdot{ }^{2} /$ nucleon between 1 and 40 $\mathrm{GeV}$.

The last session was devoted to a discussion of the results of recent experiments on low-energy muons and electrons. The general impression that emerges from studies of muon decay and capture is that whereas the decay process is fairly well understood there are still some uncertainties in the explanation of the observed capture-rates. The discussion of electron interactions centred mainly around the Stanford work on nuclear sizes. One of the most interesting results of this work is the small size of the electrostatic charge core of the neutron. The interpretation of this result is not clear and the confirmation or otherwise of this result by more accurate experiments is awaited.

The conclusions to be drawn from this rather overworked Conference appear to be that there is still much to be learned about virtually every aspect of the subject. It would appear that for some time to come cosmic ray experiments are likely to be the only source of knowledge on nuclear processes at energies greater than $20 \mathrm{GeV}$. A. W. Wolmendale

\section{COMMONWEALTH UNIVERSITIES' CONGRESS, 1958}

T HE Eighth Quinquennial Congress of the Universities of the Commonwealth, the first to be held outside the United Kingdom, was held in Montreal during the first five days of September. The Congress had been preceded by a smaller conference of executive heads of the Commonwealth Universities, held in the University of Toronto during the previous week. At that conference, some useful exchanges of view took place on some current university problems. The Montreal Congress was attended by more than 400 delegates and guests, many of them accompanied by their wives. Among the guests were some twenty-four American university presidents and the members of the administrative board of the International Association of Universities. The Congress was probably the most comprehensive gathering of representatives of the university world of the British Commonwealth and the United States which has yet taken place. There was consensus of opinion that never before had so much mutual understanding been achieved, both by the sessions of the Congress and otherwise, of the general and detailed patterns of university institutions in the countries represented at the Congress. Most of the Congress sessions were held at McGill University, although one session was held in the University of Montreal. Both universities provided accommodation and lavish hospitality for the delegates and their wives.

At the opening ceremony on September 1 , the delegates and guests were welcomed by Dr. Andrew Stewart, president of the University of Alberta, in his dual capacities of chairman of the Association of Universities of the British Commonwealth and president of the National Conference of Canadian Universities. The Congress was formally opened by the Right Hon. Vincent Massey, governor-general of Canada, who warned that universities to-day tread a dangerous path in increasingly accepting government money, offered because they are doing things that government wants done. "The intellectual duty of the university to remain free is," he insisted, "greater now than ever, and more difficult than ever before." The opening address of the Congress, on "Expanding Education", was then delivered by Sir Hector Hetherington, principal of the University of Glasgow, who described himself as the veteran who had attended all but one of the previous seven congresses. He referred to two new elements in the expansion of university education in all the countries represented at the Congress - that the required rate of expansion is abnormally high, and that what is wanted is not a general all-round expansion but one designed to take effect mainly in one direction, science and applied science. Sir Hector posed no new problems and proffered no new solutions. He explained how the present situation had come about and what steps had been taken, and were being taken, to deal with it. He propounded the principles which, in his view, needed to be kept in mind in the expansion of professional education, if it were not to become mere vocational training, and he urged the value of keeping the humanities, together with science, at the centre of the university stage.

The first ordinary session of the Congress, on September 2, was devoted to a discussion of "Who should govern University Policy ?" In this and subsequent sessions the speakers tended to outline the procedures and philosophies applicable to their own countries, and this brought to light rather unexpected degrees of divergence but there was very little attempt at synthesis ; and the Congress formulated no general conclusions. The first speaker, Dr. V. C. Fowke (Saskatchewan), was concerned primarily with internal controls over university policy, and dealt specifically with the Canadian universities. $\mathrm{He}$ directed attention to two diametrically opposed concepts of university government : one, that a university ought to be a self-governing community of scholars, and the other, that it is a business enterprise which is best governed by typically corporation methods. The written constitutions of the Canadian universities tend strongly towards the latter. The allocation of funds, the determination of priorities and the selection of personnel are all the responsibilities of lay boards which have no academic representation. Usage, however, makes wide concessions to selfgovernment and it is the academic staff who determine whom they shall teach and how they shall teach, with control of examinations. They cannot have these controls without an effective voice in financial allocations. Chancellor L. A. Kimpton (Chicago), the second opening speaker, elected to defend the American practice of giving the university administrator more power, perhaps more dignity, than is usually accorded him in the British tradition. $\mathrm{He}$ pointed out that most lay boards in the American universities have only fiscal interest and power, and he claimed that the administrator should have an important role in determining policy because of the inherent 